\title{
Self-calibrating Ultrasound-to-CT Bone Registration
}

\author{
Dean C. Barratt ${ }^{1}$, Graeme Penney ${ }^{1}$, Carolyn S.K. Chan ${ }^{1}$, Mike Slomczykowski ${ }^{2}$, \\ Timothy J. Carter ${ }^{1}$, Philip J. Edwards ${ }^{3}$, and David J. Hawkes ${ }^{1}$ \\ ${ }^{1}$ Centre for Medical Image Computing, University College London, UK \\ \{d.barratt, g.penney, carolyn.chan, t.carter, \\ d.hawkes\} @cs.ucl.ac.uk \\ ${ }^{2}$ Depuy International - a Johnson \& Johnson Company, iOrthopaedics, Leeds, UK \\ ${ }^{3}$ Department of Surgical Oncology and Technology, Imperial College London, UK
}

\begin{abstract}
We describe a new self-calibrating approach to rigid registration of $3 \mathrm{D}$ ultrasound images in which in vivo data acquired for registration are used to simultaneously perform a patient-specific update of the calibration parameters of the 3D ultrasound system. Using a self-calibrating implementation of a pointbased registration algorithm, and points obtained from ultrasound images of the femurs and pelves of human cadavers, we show that the accuracy of registration to a CT scan is significantly improved compared with a standard algorithm. This new approach provides an effective means of compensating for errors introduced by the propagation of ultrasound through soft tissue, which currently limit the accuracy of conventional methods where the calibration parameters are fixed to values determined preoperatively using a phantom.
\end{abstract}

\section{Introduction}

Freehand 3D ultrasound (US) has been proposed as an accurate and non-invasive method for intraoperative registration of bony anatomy during minimally-invasive orthopaedic, neuro- and skull-based surgery [1-3]. In this technique, an US probe is tracked by a 3D localizer and may be intuitively thought of as a non-invasive device for locating bone surfaces. This approach is an attractive alternative to standard clinical methods, which are highly invasive since they require the bone surface to be surgically exposed to allow digitization by contact with a tracked sterile pointer [4,5]. Using such methods, digitized bone surface points are matched to the corresponding surface extracted from a preoperative CT-scan or, more recently, a generic bone surface model, which is simultaneously morphed to adopt the shape of an individual patient's bone [6]. Although such invasive techniques provide an accuracy that is widely accepted to be sufficient for surgical navigation, they potentially contribute to the risk of complications associated with soft tissue trauma, such as blood loss and infection.

Fig. 1(a) illustrates a B-mode US probe with rigid attachment, which is tracked by an optical localizer to enable (freehand) 3D imaging. In order to achieve accurate bone registration using such a system, careful calibration is required to maximize the localization accuracy. Specifically, calibration is required to determine the rigid-body transformation (denoted by $\mathbf{T}_{\mathrm{PROBE} \leftarrow \mathrm{US}}$ in Fig. 1(a)) which relates the 3D co-ordinate 
systems of the US image and the tracked object attached to the US probe. Numerous methods have been described for the calibration of freehand 3D US systems using a variety of special-purpose phantoms [7], but the accuracy of these methods is fundamentally limited by differences between the propagation of US in soft tissue compared and that in a phantom. These differences effectively introduce an unknown error into the calibration parameters when the system is used in vivo, limiting the localization accuracy, and, therefore, registration accuracy achievable using the 3D US technique.

(a)
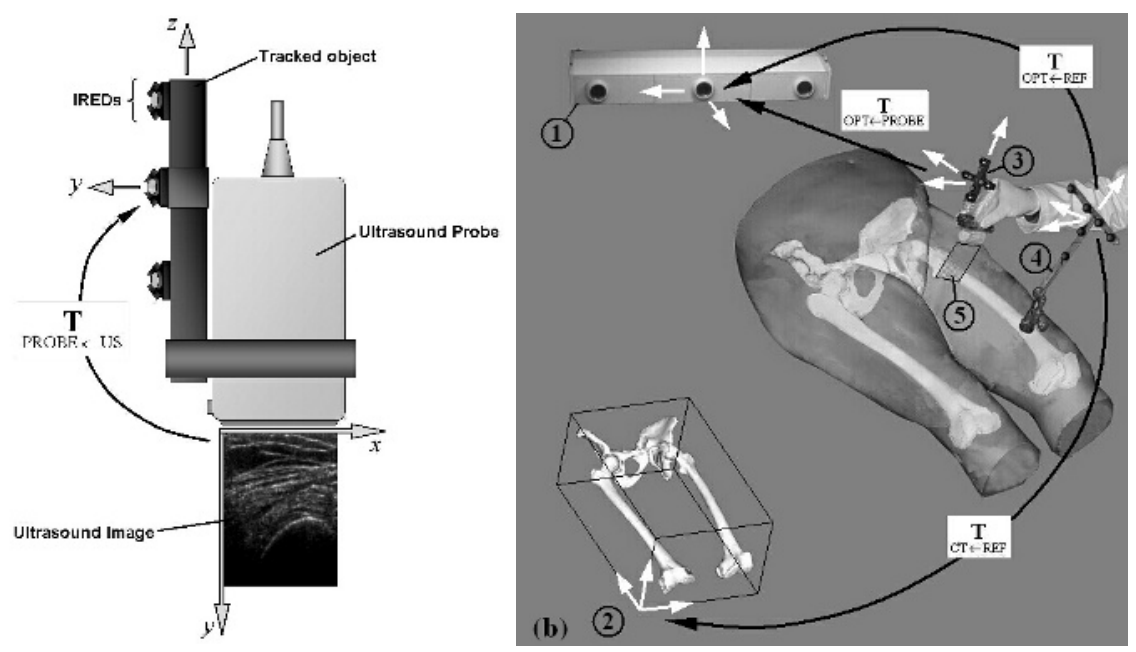

Fig. 1. (a) Left, a tracked B-mode US probe with attachment for freehand 3D US imaging. (b) Right, a schematic of the setup used for US-based registration during orthopedic surgery: (1) Optical localizer; (2) Segmented CT scan; (3) Tracked US probe; (4) Bone-implanted dynamic reference object (see text); (5) US image. The rigid-body transformations between 3D the coordinate systems of the various components are indicated by the black arrows.

A primary source of error arises from assuming a constant value for the speed of sound, typically $1540 \mathrm{~m} / \mathrm{s}$. In reality, the average speed of sound in human tissue can vary from approx. $1400 \mathrm{~m} / \mathrm{s}$ (in fat) to $1650 \mathrm{~m} / \mathrm{s}$ (in muscle), with significant variations occurring between both anatomical regions and patients. Using a simplified model of US propagation, such variations can give rise to systematic error in locating the depth of a bone surface of up to about $5 \%$, which may become particularly problematic in the obese patient. Non-linear propagation of the US beam may also become significant in some cases, potentially compromising the localization accuracy of a 3D US system further.

In the remainder of this paper we describe a self-calibrating method for US-toCT bone registration, implemented within a point-to-surface registration scheme, which addresses these issues by updating the calibration parameters of the 3D US system using in vivo data acquired for the purposes of intraoperative bone registration. 


\section{Self-calibrating Point-Based Registration Algorithm}

Fig. 1(b) shows an example set-up for hip replacement surgery in which a handheld US probe (3), tracked by an optical localizer (1), is used to obtain images of the femur and pelvis. Bone surface points derived from these images are used to calculate the rigid-body registration transformation between a tracked physical reference object (4) and a preoperative CT scan (2) by matching the US-derived points to the bone surface extracted from a CT scan. In practice, the reference object is fixed to a rigid surgical attachment implanted into a bone, as illustrated in Fig. 1(b), and is referred to as a dynamic reference object (DRO). DROs allow the patient to be repositioned during surgery without compromising the registration.

The self-calibrating, point-based registration algorithm outlined here minimizes a least-squares criterion, but differs from the popular Iterative Closest Point algorithm, in that a general non-linear optimization scheme is used to perform a least-squares minimization. If $S$ denotes the (CT-derived) bone surface, and $\left(\mathbf{p}_{n, i}\right)_{\mathrm{US}}$ is the (homogeneous) 3D position vector containing the co-ordinates of the $n^{\text {th }}$ bone surface point derived from the $i^{\text {th }}$ US image, the task of registering all points to the surface $S$ can be posed mathematically as finding the parameter vector, $\psi$, which minimizes the cost function, $C$, of the form

$$
C(\boldsymbol{\psi})=\sum_{i=1}^{I} \sum_{n=1}^{N} d^{2}\left(\mathbf{T}_{i}(\boldsymbol{\psi}) \cdot\left(\mathbf{p}_{n, i}\right)_{\mathrm{US}}, S\right),
$$

where $d(\mathbf{p}, S)$ represents the Euclidean distance between $S$ and the US point, $\mathbf{p}$, and the transformation matrix, $\mathbf{T}_{i}(\psi)$, is defined as

$$
\mathbf{T}_{i}(\boldsymbol{\psi})=\underset{\mathrm{CT} \leftarrow \mathrm{REF}}{\mathbf{T}}\left(\boldsymbol{\Psi}_{\mathrm{REG}}\right) \cdot \underset{(\mathrm{OPT} \leftarrow \mathrm{REF}, i)}{\mathbf{T}^{-1}} \cdot \underset{(\mathrm{OPT} \leftarrow \mathrm{PROBE}, i)}{\mathbf{T}} \cdot \underset{\mathrm{PROBE} \leftarrow \mathrm{US}}{\mathbf{T}}\left(\boldsymbol{\Psi}_{\mathrm{CAL}}\right) \cdot \mathbf{T}_{\mathrm{SCALE}}\left(s_{x}, s_{y}\right),
$$

where the notation $\underset{\mathrm{B} \leftarrow \mathrm{A}}{\mathbf{T}}$ denotes a rigid-body transformation from $3 \mathrm{D}$ co-ordinate system A to B. In Eqn. (2) 'US', 'PROBE', 'OPT', and 'REF' denote the 3D coordinate systems of the US image, the tracked object attached to the US probe, the (optical) 3D localizer, and the DRO, respectively (see Fig. 1(b)); $\boldsymbol{\psi}=\left[\boldsymbol{\psi}_{\mathrm{REG}}, \boldsymbol{\psi}_{\mathrm{CAL}}, s_{x}, s_{y}\right]$ is a vector containing 6 rotations, 6 translations, and 2 scaling parameters; and $\mathbf{T}_{\mathrm{SCALE}}$ is a diagonal scaling matrix included to convert the point co-ordinates from pixels to millimetres.

Conventionally, the parameters in $\psi_{\mathrm{CAL}}$ which define the transformation, $\underset{\mathrm{PROBE} \leftarrow \mathrm{US}}{\mathbf{T}}$, and the US image scaling parameters, $s_{x}$ and $s_{y}$, are determined preoperatively from one or more calibration experiments, and remain fixed during registration. However, if we consider them to be free variables, then, given a reasonable starting estimate for $\boldsymbol{\psi}$, a simultaneous registration and calibration can be carried out by optimizing all 14 parameters of $\psi$ to find the global minimum of $C$.

In our implementation, $C$ was minimized using the iterative region trust algorithm provided in the Matlab Optimization Toolbox v3.0 (The Mathworks Inc, Natick, MA, USA), and, on each iteration, point-to-surface distances were computed efficiently using a 3D Euclidean distance transform of the binary image of the bone surface generated from a pre-segmented CT scan [8]. 
In order to avoid local minima, a good initial estimate of the registration parameters $\left(\psi_{\mathrm{REG}}\right)$ was obtained by first performing a standard rigid registration with all of the calibration parameters fixed to values obtained from a prior phantom-based calibration (see Sect. 3.2 below). The performance of the self-calibrating registration algorithm was evaluated using data acquired from the pelvis and femurs of 3 complete female cadavers with intact tissue in experiments which simulated the conditions of hip replacement surgery. Importantly for this study, the use of cadavers allowed an accurate Gold Standard registration, based on bone-implanted fiducials, to be established.

\section{Evaluation of Registration Accuracy Using Cadaveric Data}

\subsection{Cadaver Preparation and CT Scanning}

With the cadavers lying in the supine position, titanium bone screws with a $2 \mathrm{~mm}$ diameter threaded tip were implanted into both femurs and each side of the pelvis. Four bone screws were inserted into each femur and 5 into each hemi-pelvis. A custommade fiducial marker filled with a radio-opaque contrast agent was then attached to the end of each screw, after which the cadavers were transported to the radiology department where a single high-resolution CT scan of each cadaver was performed using a Siemens SOMATOM Plus 5 spiral CT scanner. CT images were reconstructed with voxel dimensions between 0.7 and $0.8 \mathrm{~mm}$ in the transverse plane and a slice thickness of $2 \mathrm{~mm}$. Following CT scanning, the cadavers were returned to the anatomy laboratory and the contrast-filled fiducial markers replaced with markers containing a $3 \mathrm{~mm}$ diameter divot, machined so that the centre of a ball-tip digitizer placed into the divot coincided with the centroid of the contrast-filled chamber of the imaged markers. The digitizer, US probe, and a DRO implanted into the femur were all tracked using an Optotrak 3020 optical localizer (Northern Digital Inc., Ontario, Canada).

The Gold Standard image-to-physical registration transformation for each bone was found by registering the physical positions of the centroid of each contrast-filled fiducial marker, identified using the ball-tip digitizer, to the corresponding manuallyidentified positions in the CT scan. The surface of each bone was segmented in the CT images using a combination of manual and semi-automatic tools provided by the software package Analyze v6.0 (Mayo Foundation, Rochester, MN, USA). From the segmented CT images, a binary image which just contained the bone surface voxels was generated, and this was used to compute a 3D Euclidean distance image for the point-based registration algorithm.

\subsection{Ultrasound Imaging}

The freehand 3D US system used in this study was developed by our research group and is based on a standard clinical US scanner (Philips-ATL HDI-5000, Philips Medical Systems, Bothell, WA, USA). A high-frequency linear-array probe (L12-5 scan-probe; $5-12 \mathrm{MHz}$ broadband transducer) was chosen, which was tracked in 3D by attaching a custom-made cross-shaped piece with 20 IREDs attached as shown in Fig. 1(a). Acquisition software, also developed in our laboratory, was used to synchronize the recording of the $3 \mathrm{D}$ position and orientation of the US probe (relative to the DRO) with US image capture using an analogue-to-digital converter. 

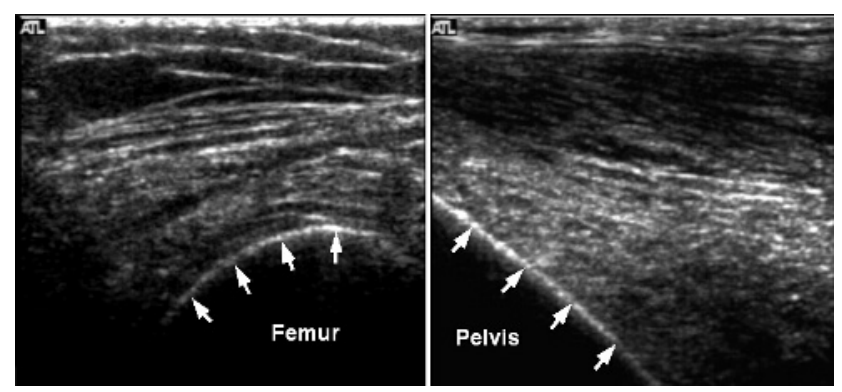

Fig. 2. Example US images of the caderveric femur (left) and pelvis (right) obtained in this study. The arrows indicate the bone surface.

To provide a reasonable starting estimate of the calibration parameters of the 3D US system, a pinhead immersed in a water-glycerol solution was scanned and the calibration parameters calculated using a previously published method [9]. The scaling of the captured US images was determined from electronic calliper measurements provided by the US scanner. During US scanning the probe was covered with a plastic sheath containing a small quantity of gel to ensure good acoustic contact. For each cadaver, B-mode US images of the surface of the femur and pelvis were acquired using realtime $2 \mathrm{D}$ compounding ("SonoCT ${ }^{\mathrm{TM}}$ ") with a single focal zone and a maximum penetration depth of $6 \mathrm{~cm}$. Example US images are shown in Fig. 2.

The bone surface was segmented manually from US images by defining a number of points on the maximum intensity of the ridge corresponding to the bone surface in each US image. Approximately 10 points were defined for each image in which the bone surface was clearly visible. Images with poor tracking data were excluded automatically by segmenting only those images where $\geq 10$ out of 20 IREDs on the US probe attachment and $\geq 5$ out of 6 IREDs on the DRO were visible to the Optotrak.

\subsection{Registration Experiments}

To test the accuracy and robustness of the US-based registration, 100 registrations were performed for each of the 9 bones ( 3 whole pelves and 6 femurs) using a random starting position for each trial. Starting positions were generated by simulating a simple intraoperative, point-based registration procedure in which the surgeon identifies a series of skin points adjacent to well-defined bony landmarks. To do this, points on the skin surface close to the landmarks were first identified by hand in each CT image. Three points were identified for each femur, near to the medial and lateral epicondyles, and to the greater trochanter. For each pelvis, 4 points were selected in the proximity of the left and right superior pubic ramus and left and right anterior superior iliac spines. To simulate errors associated with intraoperative identification of anatomical landmarks, a set of random points on the skin surface were computed, each within a sphere of radius $20 \mathrm{~mm}$ centred at the corresponding manually-selected point. These points were then transformed to the co-ordinate system of the DRO using the Gold Standard registration transformation. The maximum error of $20 \mathrm{~mm}$ was chosen to correspond to the upper limit on the error that might reasonably occur when 
landmarks are digitized manually [10]. Greater accuracy could be obtained by using self-adhesive skin markers, for example.

In the case of the femur, the estimated centre of rotation of the femoral head provided an additional anatomical point for calculating registration starting positions. The location of this point for each hip joint was determined experimentally by slowly pivoting the leg, while continuously tracking the DRO rigidly attached to the femur. A random offset of up to $10 \mathrm{~mm}$ was added to this point to simulate the variability that might reasonably be expected using the pivot method intraoperatively.

Random starting positions for the registration trials were found by registering the original set of manually-identified points in the CT image to the corresponding set of simulated anatomical points. As indicated in Sect. 2, each registration trial required an initial 2-step rigid registration with the calibration parameters fixed - once using all US points and then repeated with outliers removed - to obtain a good estimate of the registration parameters. Then, each time using these estimates as starting values, a self-calibrating registration was performed twice, once optimizing 7 parameters, including axial scaling $\left(s_{y}\right)$ as an additional free parameter, and then again with a full 14 parameter optimization. For each registration trial, starting estimates of the calibration parameters were calculated using a randomly selected subset of 30 of the 71 images of the calibration phantom to introduce variability into the starting calibration parameter estimates. In the case of the US scaling parameters, starting estimates were generated by adding a uniform random noise of up to $+/-5 \%$ to the values determined using the standard calibration method.

Registration accuracy was evaluated by calculating the target registration error (TRE) for each bone surface voxel extracted from the CT scan using the formula

$$
\operatorname{TRE}\left(\mathbf{s}_{\mathrm{CT}}\right)=\left\|\mathbf{s}_{\mathrm{CT}}-(\underset{\mathrm{CT} \leftarrow \mathrm{REF}}{\mathbf{T}})_{\mathrm{US}} \cdot\left(\begin{array}{c}
\mathbf{T}^{-1} \\
\mathrm{CT} \leftarrow \operatorname{REF}
\end{array}\right)_{\mathrm{GOLD}} \cdot \mathbf{s}_{\mathrm{CT}}\right\|,
$$

where $\mathbf{s}_{\mathrm{CT}}$ is the (homogeneous) 3D position vector of the centre of a bone surface voxel in CT co-ordinates, and the subscripts 'US' and 'GOLD' denote transformations computed using US-based and Gold Standard registrations, respectively. (Note: the magnitude operator in Eqn. (3) ignores the redundant $4^{\text {th }}$ element of the vector operand). The root-mean-square (RMS) TRE was computed for all surface voxels of each bone. As a further clinically important measure of registration accuracy, the RMS TRE was calculated using a subset of voxels within the surgical region of interest (SROI). For the femur, the SROI was defined as the region proximal to the lesser trochanter, which includes the femoral head and neck, whereas for the pelvis the SROI was defined as the spherical region enclosing the acetabulum with radius $50 \mathrm{~mm}$ and centred at the approximate centre of the femoral head.

\section{Results}

The predicted TREs for the Gold Standard registrations, calculated over the whole bone surface using the method of Fitzpatrick et al. [11], were submillimetre in all but one case (range: 0.13 to $1.32 \mathrm{~mm}$ RMS), indicating excellent Gold Standard 
registrations. The number of US images acquired was between 257 and 576, with between 124 and 510 images segmented, giving a total of between 960 and 6112 surface points for each bone ( 10 points per US image).

Table 1. Average RMS TRE (absolute maximum TRE in brackets) in millimetres for US-to-CT bone registration from 100 trials of 9 datasets. Key: The "Initial TRE" results from the registration based on simulated anatomical landmarks, identified intraoperatively; "TRE1" is the TRE for a standard rigid registration using all available US points; "TRE 2" is the TRE after removing outliers and repeating the first registration using the remaining $90 \%$ of the original points; "TRE 3" is the TRE for a 7-parameter self-calibrating registration; and "TRE 4" is the TRE for a full 14-parameter self-calibrating registration.

\begin{tabular}{llllll}
\hline \multicolumn{1}{c}{ Region } & Initial TRE & TRE 1 & TRE 2 & TRE 3 & TRE 4 \\
\hline Whole Bone & $10.25(64.88)$ & $2.57(9.30)$ & $2.20(7.37)$ & $1.87(7.15)$ & $1.59(7.15)$ \\
Surgical ROI & $9.07(39.22)$ & $2.66(6.89)$ & $2.36(7.16)$ & $1.97(5.13)$ & $1.74(5.44)$ \\
\hline
\end{tabular}

The average RMS TREs for each US-based registration are summarized in Table 1. It can be seen from the results presented that, overall, the self-calibrating registration resulted in a marked improvement in registration accuracy (using the KolmogorovSmirnov test, the differences between TREs 1 to 4 were found to be statistically significant at the $1 \%$ level). In particular, performing a full 14-parameter selfcalibrating registration (TRE 4) resulted in a reduction in TRE for the whole bone of approximately $28 \%$ compared with a standard point-based registration with automatic outlier removal (TRE 2). With a failed registration defined as one for which the RMS TRE over the whole bone surface was $>5 \mathrm{~mm}$, the number of failed registrations using a full self-calibration (TRE 4) was 46 from 900 trials (compared with 44 for the standard registration with outliers removed (TRE 2)) . However, of these, 39 occurred for one pelvis, resulting in a very low failure rate $(<1 \%)$ if the results from this dataset are not considered. We believe that the reason for the relatively high number of failures for this dataset is likely to be due to the much higher initial TREs (Mean = 20.07mm RMS) compared with the other datasets.

\section{Discussion and Conclusions}

In this paper we have demonstrated that optimizing the 3D US calibration parameters improves the accuracy of US-to-CT bone registration compared with a standard algorithm where calibration parameters are fixed. For the purposes of this study, manually segmented US/CT data and a point-based registration scheme were adopted, but future work will focus on developing a self-calibrating image-intensity-based registration algorithm to allow fully automatic registration, and devising an optimized clinical protocol for intraoperative US imaging of bone.

Including calibration parameters into the registration optimization provides a useful method for compensating for unpredictable, patient-specific localisation errors introduced when imaging through soft tissue. Provided fairly accurate starting 
estimates for the calibration parameters are available, updating the calibration parameters during registration also has the practical advantage that US scanner settings could be adjusted for individual patients within the operating theatre without compromising calibration or registration accuracy.

\section{References}

1. Lavallée, S., Troccaz, J., et al.: Computer-assisted spinal surgery using anatomy-based registration. In Computer Taylor, R.H., Lavallée, S., Burdea, G.C., Mösges, R. (eds): Integrated Surgery: Technology and Clinical Applications. MIT Press, Cambridge MA (1996) 425-449

2. Amin, D., Kanade, T., DiGioia, A., Jaramaz, B.: Ultrasound registration of the bone surface for surgical navigation. Comp. Aid. Surg. 8 (2003) 1-16

3. Amstutz, C., Caversaccio, M., et al.: A-mode ultrasound-based registration in computeraided surgery of the skull. Arch. Otolaryngol. Head Neck Surg. 129 (2003)1310-1316

4. DiGioia, A.M., Jaramaz, B., et al.: The Otto Aufranc Award. Image guided navigation system to measure intraoperatively acetabular implant alignment. Clin. Orthop. 355 (1998) 8-22

5. Ma, B. and Ellis, R.B.: Robust registration for computer-integrated orthopedic surgery: laboratory validation and clinical experience. Med. Image Anal. 7 (2003) 237-250

6. Stindel, E., Briard, J., et al.: Bone morphing: 3D morphological data for total knee arthroplasty. Comp. Aid. Surg. 7 (2002) 156-168

7. Mercier, L., Lango, T., Lindseth, F., Collins, L.D.: A review of calibration techniques for freehand 3-D ultrasound systems. Ultrasound Med. Biol. 31 (2005) 143-165

8. Fitzgibbon, A.: Robust registration of 2D and 3D point sets. Proc. B.M.V.C. (2001) 411420

9. Barratt, D., Davies, A., et al.: Accuracy of an electromagnetic three-dimensional ultrasound system for carotid artery imaging. Ultrasound Med. Biol. 27 (2001) 1421-1425

10. della Croce, U., Cappozzo, A., Kerrigan, D.: Pelvis and lower limb anatomical calibration precision and its propagation to bone geometry and joint angles. Med. Biol. Eng. Comput. 37 (1999) 155-161

11. Fitzpatrick, M., West, J., Maurer, C.: Predicting error in rigid-body point-based registration. IEEE Trans. Med. Imag. 17 (1998) 694-702 